\title{
Photometry of 2004 RZ164: a probable binary asteroid
}

\author{
T. Kwiatkowski ${ }^{1}$, A. Kryszczyńska ${ }^{1}$, A. Marciniak ${ }^{1}$, W. Borczyk ${ }^{1}$, G. Masi², A. Galád ${ }^{3,4}$, R. Goncalves ${ }^{5}$, and F. Colas ${ }^{6}$ \\ 1 Astronomical Observatory, Adam Mickiewicz University, Słoneczna 36, 60-286 Poznań, Poland \\ e-mail: tkastr@vesta.astro.amu.edu.pl \\ 2 Department of Physics, University of Rome Tor Vergata, via della Ricerca Scientifica 1, 00133 Rome, Italy \\ 3 Modra Observatory, Department of Astronomy, Physics of the Earth, and Meteorology, FMPI, Comenius University, \\ 84248 Bratislava, Slovakia \\ 4 Astronomical Institute AS CR, 25165 Ondřejov, Czech Republic \\ 5 Instituto Politecnico de Tomar, Area de Fisica, 2300-313 Tomar, Portugal \\ 6 Institut de Mécanique Céleste, 77 Av. Denfert Rochereau, 75014 Paris, France
}

Received 18 April 2006/ Accepted 27 September 2006

\section{ABSTRACT}

\begin{abstract}
Aims. We observed a near-Earth asteroid 2004 RZ164 to study its rotational properties. The object was chosen because of its small diameter of about $700 \mathrm{~m}$, which is close to the $150 \mathrm{~m}$ threshold, separating monolithic and rubble-pile asteroids.

Methods. With small telescopes at five observatories, equipped with CCD cameras, we obtained seven lightcurves, which were taken through the clear, $R$, and $I$ filters.

Results. Despite significant changes in the observing geometries (and large phase angles during observations), the peak-to-peak amplitudes of the lightcurves never exceeded 0.15 , suggesting the almost spheroidal shape of this asteroid. There is a signature of a probable occultation/eclipse event in one of the lightcurves, which suggests 2004 RZ164 is a binary asteroid. Its primary component spins with a period of $P=2.5559 \pm 0.0001$ and the orbital period of the system is longer than 10.8 .
\end{abstract}

Key words. techniques: photometric - minor planets, asteroids

\section{Introduction}

The population of Near Earth Asteroids (NEAs) constitutes a sample of the small diameter Main Belt Asteroids (MBAs) that were redirected towards the inner parts of the Solar System. Being relatively close to the Earth, they can easily be studied by optical telescopes, radar, and space probes. Currently (spring 2006), almost 4000 of these objects have been catalogued with 800 of them being larger than $1 \mathrm{~km}$ in diameter. Investigation of NEAs is motivated by the fact that they are important in the understanding of the formation of planetesimals in the protoplanetary nebula as well as the collisional evolution of the asteroid belt. Of particular importance here are objects with diameters close to the $150 \mathrm{~m}$ threshold, separating monolithic and rubble-pile asteroids.

One of NEAs is the Apollo type 2004 RZ164, discovered by the Siding Spring Survey in Australia on 13 September 2004. Its absolute magnitude of is $H=19^{\mathrm{m}}$, which, with the assumed value of albedo ranging from 0.25 down to 0.05 , implies a diameter of $420 \mathrm{~m}$ to $940 \mathrm{~m}$, respectively. It is larger than the $150 \mathrm{~m}$ threshold, which suggests 2004 RZ164 should be a gravitationally bound rubble-pile.

On 8 December, 2004 RZ164 made a close approach to the Earth at a distance of $0.018 \mathrm{AU}$. At that time it was scheduled to be observed by a Goldstone radar, but due to the extension of the maintenance/upgrade period, the observation had to be cancelled. The only possibility to learn more about this object was to observe it photometrically. The results of such observations are presented in this paper.

\section{Observations}

We observed 2004 RZ164 on 7 and 10 Dec. (Linhaceira, Portugal), 15 Dec. (Tenagra, USA), 15 and 16 Dec. (Borowiec, Poland), 21 Dec. 2004 (Modra, Slovakia), and 4 Jan. 2005 (Pic du Midi, France) collecting 7 lightcurves (Table 1). During that time the asteroid swept a long arc in the sky, while its solar phase angle was always in the range from $38^{\circ}$ to $50^{\circ}$. All the lightcurves are displayed in Fig. 1 after being arbitrary shifted in time and magnitude to fit in one plot.

The first photometric data on 2004 RZ164 were collected at the privately owned Linhaceira observatory (IAU code 938), with the Meade LX-200 Schmidt-Cassegrain telescope $(D=$ $0.25 \mathrm{~m}, F / 5.8)$ equipped with the MX916 Starlight Xpress CCD camera $(752 \times 580$ Sony ICX083AL chip), binned at $2 \times 2$. Due to the small aperture of the telescope, no filter was used during observations. A rapid movement of the asteroid on the sky prompted very short exposure times of 6-10 s. The CCD frames were reduced with the WinAstrometrica program and the profile fitting photometry was performed on the stacks of 3 images of $6 \mathrm{~s}$ each $(7 \mathrm{Dec}$.$) or on single 10 \mathrm{~s}$ images $(10 \mathrm{Dec}$.$) . The ob-$ tained photometric measurements were then averaged to form the final lightcurve.

On 15 Dec. 2004 RZ164 was observed at the $0.81 \mathrm{~m}$ Tenagra II robotic telescope located in Sonoran desert, Arizona, USA. It is a F/7 reflector, equipped with a Apogee AP8 CCD camera $(1 \mathrm{k} \times 1 \mathrm{k}$ SITe SI003 chip). At that time the asteroid slowed down so that the $R$ filter $60 \mathrm{~s}$ exposures could be used without trailing its image. The frames were reduced with the MaxIM DL program and the aperture photometry was used to get the relative magnitudes of the asteroid. 
Table 1. Aspect data for 2004 RZ164.

\begin{tabular}{ccccccc}
\hline \hline $\begin{array}{c}\text { Date } \\
\text { 2004/2005 }\end{array}$ & $r$ & $\Delta$ & $\begin{array}{c}\text { Phase } \\
\text { angle }\end{array}$ & \multicolumn{2}{c}{$\lambda(\mathrm{J} 2000)$} & \\
$(\mathrm{UT})$ & $(\mathrm{AU})$ & $(\mathrm{AU})$ & \multicolumn{1}{c}{$\left(^{\circ}\right)$} & \multicolumn{1}{c}{$\left.{ }^{\circ}\right)$} & \multicolumn{1}{c}{$\left.{ }^{\circ}\right)$} & \\
\hline Dec. 07.8 & 0.997 & 0.018 & 49.9 & 25.4 & -2.4 & Lin \\
Dec. 10.9 & 1.004 & 0.025 & 37.5 & 67.1 & 36.7 & Lin \\
Dec. 15.4 & 1.019 & 0.052 & 47.1 & 98.3 & 47.6 & Ten \\
Dec. 15.9 & 1.020 & 0.055 & 47.4 & 100.1 & 47.8 & Bor \\
Dec. 16.9 & 1.024 & 0.061 & 48.0 & 103.2 & 48.2 & Bor \\
Dec. 22.0 & 1.041 & 0.089 & 48.1 & 110.9 & 48.6 & Mod \\
Jan. 04.9 & 1.121 & 0.192 & 40.8 & 118.5 & 46.6 & Pic \\
\hline
\end{tabular}

Observatory Code: Bor - Borowiec; Pic - Pic du Midi; Lin Linhaceira; Mod - Modra.

Almost at the same time, the asteroid was observed at the Borowiec Observatory in Poland. The $0.4 \mathrm{~m}$ Newtonian telescope, equipped with the SBIG ST-7 CCD camera, collected about five times fewer photons than that at Tenagra, and so to obtain the same $\mathrm{S} / \mathrm{N}$, exposure time was set to $300 \mathrm{~s}$ and no filter was used. This setup, however, produced trailed images of the asteroid and so the telescope tracking rate was changed to follow 2004 RZ164. This was easy to do because on 15 and 16 Dec. the asteroid moved parallel to the equator. CCD frames were reduced with a CCLRC STARLINK package, as is described in Michalowski et al. (2004).

A relatively bright NEA drew the attention of the observers at the Modra Observatory in Slovakia, who observed it on 21 Dec. with a $0.6 \mathrm{~m}, \mathrm{~F} / 5.5$ reflector, equipped with a Apogee AP8p CCD camera $(1 \mathrm{k} \times 1 \mathrm{k}$ SITe SI003 sensor) located at primary focus. The asteroid was observed without a filter with $60 \mathrm{~s}$ integration time. The standard bias, dark, and flat field corrections as well as an aperture photometry was done with the Maxim DL program.

A surprising, almost constant amplitude of 2004 RZ164 light variations prompted us to observe it once more at the Pic du Midi Observatory (France). A $1.05 \mathrm{~m}$ telescope equipped with the $384 \times 288$ Thomson THX 7863 CCD camera was used to obtain a short, rather noisy lightcurve. The individual frames were exposed for $240 \mathrm{~s}$ through the Schott $R G 695$ filter, which, combined with the spectral response of the CCD sensor, is similar to the standard $I$ filter. To provide similar image profiles for both the asteroid and comparison stars, the tracking of the telescope was set to half of the asteroid on-the-sky movement. After the bias and flat field correction with the ASTROL package (developed at IMCCE) the asteroid brightness was measured with the PHOTOM aperture photometry programme included in the CCLRC STARLINK package.

\section{Signs of binarity of 2004 RZ164}

Despite large solar phase angles during observations of 2004 RZ164, the amplitudes of its lightcurves never exceeded 0.15 mag (Fig. 1). Small lightcurve amplitudes at high solar phase angles mean that the asteroid is almost spheroidal, or that it is elongated, but observed almost pole-on. The latter was true, for example, for a cigar-shaped 433 Eros on 4 Oct. 1951 or 22 and 25 Aug. 1974, when its lightcurve amplitude was only $0.1 \mathrm{mag}$. At that time the solar phase angles of Eros were $\alpha \approx 20^{\circ}$ and $\alpha \approx 40^{\circ}$, respectively. In the case of 2004 RZ164, however, we observed it at several different positions in the sky, which favor the former explanation: the almost spherical shape of the rotating asteroid.
Light variations of nearly spheroidal asteroids, observed at high solar phase angles, are more influenced by minor irregularities of their shapes than by the general elongation of the body. It is therefore quite common to observe more complicated lightcurves in such cases, sometimes with three maxima and three minima. In the lightcurves of 2004 RZ164, however, it is difficult to notice a pattern that repeats itself. This is best visible in the 15 and 16 Dec. data, which span 5-7 h each and being taken at the same position in the sky - should make a nice composite lightcurve. Unfortunately, trials with different periods gave poor results and there were clear outliers that could not be brought in line with the rest of the points. This usually means either a non-principal axis rotation of the asteroid or a binary system in which rotations of both components contribute to the observed light variations.

The latter explanation is supported by an independent analysis of part of the data done by Pravec (2006). He first pointed out that a strange drop in the asteroid brightness during the 24 Dec. Modra run could not be an artifact - something we believed at first - but the beginning of the attenuation event in the binary system. A careful reanalysis of the data showed the effect is a real one, and we do have a sign of binarity of 2004 RZ164. The lack of points immediately after the drop of brightness was caused by temporarily switching the telescope to another object - a rather unfortunate decision given the importance of this part of the lightcurve.

As four of our lightcurves (Tenagra 15 Dec., Borowiec 15, and 16 Dec., and Modra 21 Dec.) were obtained within a relatively short interval of 6 days during which the observing geometry was almost the same, we can safely assume that we should be able to detect another attenuation of such magnitude if it happened during the observations. The lack of it places some limits on the orbital period $P_{\text {orb }}$ of the secondary component. Assuming that:

- the drop in the asteroid brightness detected in the Modra lightcurve was a beginning of the attenuation;

- the attenuation lasted 54 min (from JD 2453361.3927 till 2453361.4300 ), which is a conservative assumption in comparison to other binary asteroids (see, for example, Pravec et al. 2006),

- both primary and secondary eclipses/occultations in this system are of sufficient depth to be easily detected; and

- the secondary eclipse happens at orbital phases between 0.4 and 0.6 (which is the case if the orbit is not circular),

it can be found (by checking different values for $P_{\text {orb }}$ ) that the orbital period should be longer than $21 \mathrm{~h} .8$. However, if the third assumption is not valid and the secondary eclipse is so shallow that it could not be clearly visible during one of the observations, the constraint on $P_{\text {orb }}$ is much weaker, and any value longer than $10^{\mathrm{h}} .8$ could be possible. This value is in good agreement with the lower cut-off for the orbital periods of the binary near-Earth asteroids, found by Pravec et al. (2006). Examining 17 systems they concluded that for all of them, $P_{\text {orb }}$ was longer than about $11^{\mathrm{h}}$.

\section{Rotation of primary component}

Assuming the observed light variations of 2004 RZ164 are caused by two bodies orbiting each other, we searched for the presence of the additional periodicities connected with the rotation of the secondary component. For that we performed a Fourier analysis of the four best lightcurves (from Tenagra, 


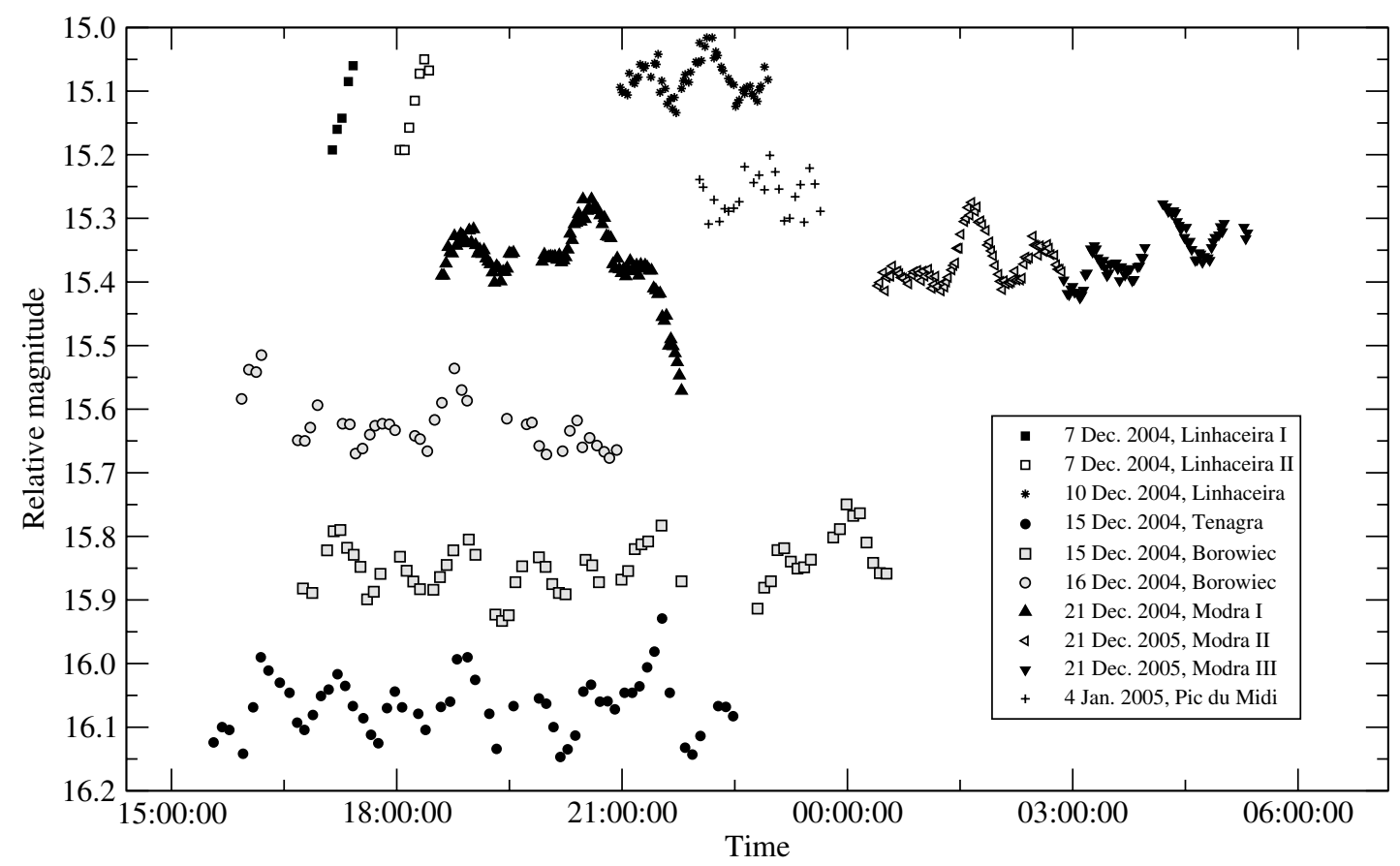

Fig. 1. Lightcurves of 2004 RZ164, arbitrarily shifted in time and brightness to fit them in one plot. All magnitudes are relative and unfiltered except the Tenagra ( $R$ filter) and Pic du Midi ( $I$ filter) data. During two nights, 7 and 21 Dec. 2004, the comparison stars changed during observations making it difficult to connect parts of the lightcurve in magnitude - these parts are marked with different symbols. Note the drop in the asteroid brightness in the Modra I lightcurve probably caused by the beginning of the eclipse/occultation event.

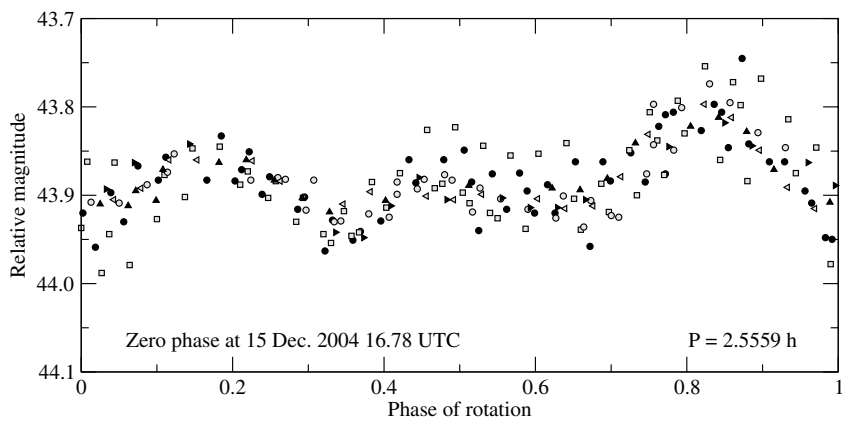

Fig. 2. Composite lightcurve of 2004 RZ164, made from the data from Tenagra (15 Dec.), Borowiec (15 and 16 Dec.), and Modra (21 Dec.) observatories. The symbols are the same as in Fig. 1. Part of the Modra lightcurve showing the attenuation was removed. Also, the density of points in the Modra lightcurve was brought to the same level as in the rest of lightcurves by averaging them by groups of four.

Borowiec, and Modra) using the code by Schwarzenberg-Czerny (private communication), which is optimized for the unevenly spaced data. Unfortunately, all of the lightcurves were on the relative magnitude scale, so to analyze them simultaneously we had to compute the mean value of each of them and assume it to be zero. This way we lost the information on periods longer than the longest lightcurve in our sample.

The strongest frequency in the power spectrum (Fig. 3) is $f_{3}=28.17$, but there are also two other distinct frequencies, $f_{1}=$ 9.38 and $f_{2}=18.78$ whose sum gives us almost exactly $f_{3}$. The rest of the visible peaks were identified as aliases. During many trials it appeared that a subtraction of a Fourier series of the third order and a frequency $f=9.38$ from the asteroid lightcurves removed all signal from the power spectrum down to the noise level. This made us certain that, despite previous problems with making a composite lightcurve, that all periodicity - at least in

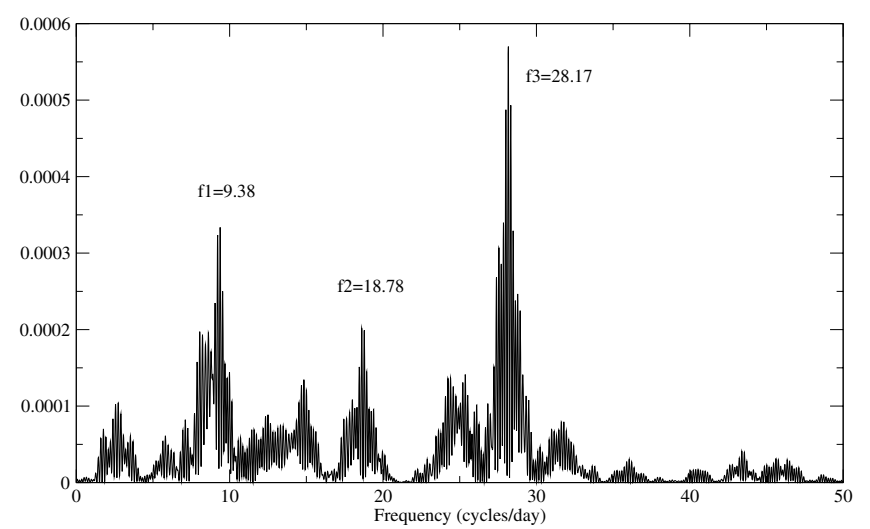

Fig. 3. Power spectrum of the Tenagra, Borowiec, and Modra lightcurves. The $f_{1}$ and $f_{2}$ frequencies are in fact pairs of close peaks: $9.22,9.38$ in the case of $f_{1}$, and 18.61 and 18.78 in the case of $f_{2}$. The other lower peaks are aliases.

the four lightcurves - comes from a single lightcurve with three maxima and three minima and a period of $P=2.5559$.

Knowing this we constructed a new composite lightcurve with a period of $P=2$ h.5559, which gave us a curve with three maxima and three minima per rotation (Fig. 2). Its poor quality is probably caused by the light of the secondary component, whose light variations left no detectable trace in the power spectrum. This can happen if the rotation of the secondary is synchronized with its orbital motion and thus lost in the arbitrary shifts introduced to bring all lightcurves to the same magnitude level.

\section{Conclusions}

From the photometric observations performed during the Dec.Jan. apparition of 2004 RZ164, we can conclude that this asteroid is a probable binary system in which the almost 
spheroidal primary component rotates with a period $P=$ $2.5559 \pm 0.0001$ and the orbital period of the secondary component is longer than $10^{\mathrm{h}} 8$. It is quite possible that the rotation of the secondary component is synchronized with its orbital motion. During the next 10 years, 2004 RZ164 will not be brighter than $20^{\mathrm{m}}$ to the observer on Earth, which makes further studies of this object very difficult. This emphasizes the importance of well coordinated observing campaigns in studying these small yet interesting objects shortly after their discovery.

Acknowledgements. We would like to thank L. Kornoš and J. Világi from the Modra Observatory and F. Mallia from the Campo Catino Observatory for their help with the observations. The first three authors were supported by the Polish KBN Grant 2 P03D 007 22, while the sixth author acknowledges support from both the Slovak Grant Agency for Science VEGA (grant 1/3074/06) and by the Grant Agency of the Czech Republic (grant 205/05/0604). The Borowiec observations were reduced with the CCLRS STARLINK package.

\section{References}

Michalowski, T., Kwiatkowski, T., Kaasalainen, M., et al. 2004, A\&A, 416, 353 Pravec, P. 2006, Prepublished periods of NEAs, data file retrived 1 Feb. 2006 http://sunkl.asu.cas.cz/ ppravec/newres.txt

Pravec, P., Scheirich, P., Kušnirák, P., et al. 2006, Icarus, 181, 63 